\title{
Theoretical Analyses of Superconductivity in Iron Based Superconductor
}

$$
\mathrm{Ba}_{1-\mathrm{x}} \mathrm{K}_{\mathrm{x}} \mathrm{Fe}_{2} \mathrm{As}_{2}
$$

Tadesse Desta $^{1^{*}}$, Gebregziabher Kahsay ${ }^{2}$ and Pooran Singh ${ }^{3}$

${ }^{1}$ Department of Physics, Jigjiga University, Jigjiga, Ethiopia (*tad4aau@ @mail.com)

${ }^{2}$ Department of Physics, College of Science, Bahir Dar University, Bahir Dar, Ethiopia

${ }^{3}$ Department of Physics, Addis Ababa University, Addis Ababa, Ethiopia

\begin{abstract}
This paper focuses on the theoretical analysis of superconductivity in iron based superconductor $\mathrm{Ba}_{\mathrm{x}-1} \mathrm{~K}_{\mathrm{x}} \mathrm{Fe}_{2} \mathrm{As}_{2}$. After reviewing the current findings on this system, we suggest that phononexciton combined mechanism gives a right order of superconducting transition temperature $\left(\mathrm{T}_{\mathrm{C}}\right)$ for $\mathrm{Ba}_{1-x} \mathrm{~K}_{x} \mathrm{Fe}_{2} \mathrm{As}_{2}$. By developing a model Hamiltonian for the system under consideration, using double time temperature dependent Green's function formalism and a suitable decoupling approximation technique, we have analyzed theoretically the superconductivity of iron-based superconductor $B a_{1-x} K_{x} F e_{2} A s_{2}$. Furthermore, by using the experimental and plausible theoretical values of the parameters in the obtained expressions, phase diagrams of superconducting transition temperature $\left(T_{\mathrm{C}}\right)$ versus electron coupling constant $(\lambda e)$ and superconducting transition temperature $\left(T_{\mathrm{C}}\right)$ versus superconducting gap parameter $(\Delta)$ have been plotted. Our findings demonstrate that, as the electron coupling constant $(\lambda e)$ increases the superconducting transition temperature $\left(\mathrm{T}_{\mathrm{C}}\right)$ increases and vice versa and also as the temperature increases the superconducting order parameter decreases and vanishes at the transition temperature $\left(\mathrm{T}_{\mathrm{C}}\right)$ for $B a_{1-x} K_{x} F e_{2} A s_{2}$. Our findings are in a broad agreement with the experimental observations.
\end{abstract}

Keywords: Iron based Superconductors, Green's Function formalism, Superconducting order parameter, $\mathrm{Ba}_{1-\mathrm{x}} \mathrm{K}_{\mathrm{x}} \mathrm{Fe}_{2} \mathrm{As}_{2}$.

\section{INTRODUCTION}

Superconductivity is a remarkable macroscopic quantum phenomenon, which was discovered by Onnes (1911) while investigating the electrical resistivity of mercury at low temperature. Onnes (1911) found that, when mercury was cooled below $T_{C}=4.2 \mathrm{~K}$, the resistivity abruptly dropped to a value close to zero and the material became a superconductor. The discovery of the phenomenon of zero electric resistivity in mercury was soon followed by the observation of the superconducting state in other materials. The microscopic understanding of the phenomenon, however, was not given due attention until the formulation of the superconductivity theory by Bardeen, Cooper and Schrieffer in 1957(BCS) who provided a microscopic theoretical analysis suitable for describing the observed superconducting state (Bardeen et al., 1957). As is well known, the core point of the BCS theory is the formation of Cooper pairs. Superconducting 
materials are characterized by the presence of mainly two characteristic effects, the effect of zero electrical resistivity and the diamagnetism or the Meissner effect, which is the complete expulsion of magnetic field from the interior of a given superconducting material for temperatures below the critical temperature $\left(\mathrm{T}_{\mathrm{C}}\right)$. Furthermore, there was a great excitement after the discovery of high temperature superconductor cuprate by Bednorz and Müller (1986).

The recent discovery of superconductivity in iron-pnictides with transition temperature, $\mathrm{T}_{\mathrm{C}}=$ 26K (Kamihara et al., 2008) has generated enormous interest in the study of these superconductors. This discovery has provided an excellent opportunity to understand the behavior of unconventional or low temperature superconductors. After the discovery of iron based superconductors by Kamihara et al. (2008) different compounds were discovered by replacing lanthanum by magnetic rare earth elements such as $\mathrm{Ce}, \mathrm{Sm}, \mathrm{Nd}$ or $\mathrm{Pr}$ and the critical temperature could be easily raised up to 56K (Wang et al., 2008a). In iron based superconductors (IBSC), superconductivity usually occurs by doping either electrons or holes in the FeAs layers. The iron, $\mathrm{Fe}^{2+}$ forms tetrahedron within the layers. This means that, ironpnictide Fermi level is formed by $3 d_{x y}, 3 d_{y z}$, or $3 d_{z x}$ orbitals. Very intriguing property of the IBSC is rather high flexibility concerning elemental substitution, leading to the formation of several families of iron based superconductors. Based on their chemical composition, iron based superconductors are categorized as 1111 family (Kamihara et al., 2008), 122 family (Rotter et al., 2008), 111 family (Wang et al., 2008b) and 11 family (Hsu et al., 2008). These newly discovered iron based layered pnictides have triggered challenges towards understanding of their pairing mechanisms. Superconductivity in iron-pnictides appears by doping the parent compounds with charge carriers. Furthermore, there is a resistivity anomaly in the parent compounds of $\mathrm{LaFeAsO}$ at around $150 \mathrm{~K}$ which disappears as superconductivity emerges in addition to a small anomaly in the dc magnetic susceptibility. Optical conductivity and theoretical calculations suggest that, $\mathrm{LaFeAsO}$ exhibits a spin density wave (SDW) instability, that is, suppressed by doping with electrons to induce superconductivity (Kamihara et al., 2008).

\section{MODEL HAMILTONIAN}

In order to obtain an expression for superconducting transition temperature $\left(\mathrm{T}_{\mathrm{C}}\right.$ ) and superconducting order parameter $(\Delta)$ from exclusively phonon-exciton combined mechanism, we 
calculated $\mathrm{T}_{\mathrm{C}}$, reduced gap $\left(2 \Delta / k_{B} T_{C}\right)$ and compared the values with the experimental one for the Iron based superconductor $\mathrm{Ba}_{1-\mathrm{x}} \mathrm{K}_{\mathrm{X}} \mathrm{Fe}_{2} \mathrm{As}_{2}$.

The Hamiltonian of a system of conduction electrons interacting with phonons (phonon-exciton) can be described by the two level systems as follows (Singh and Sinha, 1990); and (Allender et al., 1973).

$\widehat{\mathrm{H}}=\widehat{\mathrm{H}}_{\mathrm{el}}+\widehat{\mathrm{H}}_{\mathrm{ph}}+\widehat{\mathrm{H}}_{\mathrm{el}-\mathrm{ph}}+\widehat{\mathrm{H}}_{\mathrm{c}}$

Where, $\widehat{\mathrm{H}}_{1}=\sum_{\kappa, \sigma} \epsilon_{\kappa} \hat{\mathrm{a}}_{\kappa, \sigma}^{\dagger} \hat{\mathrm{a}}_{\kappa, \sigma}$ and is the Hamiltonian or energy of mobile (conduction) electrons, $\hat{\mathrm{a}}_{\kappa, \sigma}^{\dagger}\left(\hat{\mathrm{a}}_{\kappa, \sigma}\right)$ are the creation (annihilation) operators for conduction electrons with the wave vector $\mathbf{k}$ and spin projection in the $\mathrm{z}$-axis, $\sigma=\uparrow$ or $\downarrow$. $\epsilon_{\mathrm{K}}$ is the single electron kinetic energy.

$\widehat{\mathrm{H}}_{\mathrm{ph}}$ is the standard phonon Hamiltonian, which can be written as,

$\widehat{\mathrm{H}}_{\mathrm{ph}}=-\sum_{\kappa, \kappa^{\prime}} \mathrm{V}_{\mathrm{ph}} \hat{\mathrm{a}}_{\kappa \uparrow}^{\dagger} \hat{\mathrm{a}}_{-\kappa \downarrow}^{\dagger} \hat{\mathrm{a}}_{\kappa^{\prime} \downarrow} \hat{\mathrm{a}}_{-\kappa^{\prime} \uparrow}$

$\widehat{\mathrm{H}}_{\mathrm{el}-\mathrm{ph}}$ is the electron-phonon interaction Hamiltonian and is expressed as,

$\widehat{\mathrm{H}}_{\mathrm{el}-\mathrm{ph}}=-\sum_{\kappa, \kappa^{\prime}} \mathrm{V}_{\mathrm{el}} \hat{\mathrm{a}}_{\kappa \uparrow}^{\dagger} \hat{\mathrm{a}}_{-\kappa \downarrow}^{\dagger} \hat{\mathrm{a}}_{\kappa^{\prime} \downarrow} \hat{\mathrm{a}}_{-\kappa^{\prime} \uparrow}$

$\widehat{\mathrm{H}}_{\mathrm{c}}=\sum_{\kappa, \kappa^{\prime}} \mathrm{V}_{\mathrm{c}} \hat{\mathrm{a}}_{\kappa \uparrow}^{\dagger} \hat{\mathrm{a}}_{-\kappa \downarrow}^{\dagger} \hat{\mathrm{a}}_{\kappa^{\prime} \downarrow} \hat{\mathrm{a}}_{-\kappa^{\prime} \uparrow}$

and is the screened Coulomb interaction between conduction electrons.

Thus, using equations (2-4) in equation (1), we obtain,

$\widehat{H}=\sum_{\kappa, \sigma} \epsilon_{\kappa} \hat{a}_{\kappa, \sigma}^{\dagger} \hat{a}_{\kappa, \sigma}-\sum_{\kappa, \kappa^{\prime}}\left(V_{p h}+V_{e l}-V_{c}\right) \hat{a}_{\kappa \uparrow}^{\dagger} \hat{a}_{-\kappa \downarrow}^{\dagger} \hat{a}_{\kappa^{\prime} \downarrow} \hat{a}_{-\kappa^{\prime} \uparrow}$

\subsection{Equation of Motion for phonon-exciton Combined Mechanism}

Now, let us evaluate the following commutation relation,

$\left[\hat{a}_{\kappa \uparrow}, \widehat{H}\right]=\left[\hat{a}_{\kappa \uparrow}, \widehat{H}_{e l}\right]+\left[\hat{a}_{\kappa \uparrow}, \widehat{H}_{p h}\right]+\left[\hat{a}_{\kappa \uparrow}, \widehat{H}_{e l-p h}\right]+\left[\hat{a}_{\kappa \uparrow}, \widehat{H}_{c}\right]$

From which we obtain,

$\left[\hat{a}_{\kappa \uparrow}, \widehat{H}_{e l}\right]=\left[\hat{a}_{\kappa \uparrow}, \sum_{p, \sigma} \epsilon_{p} \hat{a}_{p, \sigma}^{\dagger} \hat{a}_{p, \sigma}\right]=\epsilon_{\kappa} \hat{a}_{\kappa, \uparrow}$

Following similar procedure as above, we get,

$\left[\hat{a}_{\kappa \uparrow}, \widehat{H}_{p h}+\widehat{H}_{e l-p h}+\widehat{H}_{c}\right]=-\sum_{k k^{\prime}}\left(V_{p h}+V_{e l}-V_{c}\right) \hat{a}_{-\kappa \downarrow}^{\dagger} \hat{a}_{k^{\prime} \downarrow} \hat{a}_{-k^{\prime} \uparrow}$

But the Greens function equation of motion is given by (Zubarev, 1960),

$\omega \ll \hat{a}_{\kappa \uparrow}, \hat{a}_{\kappa^{\prime} \downarrow}^{\dagger} \gg_{\omega}=\delta_{\kappa \kappa^{\prime}}+\ll\left[\hat{a}_{\kappa \uparrow}, \widehat{H}\right] ; \hat{a}^{\dagger}{ }_{\kappa \uparrow} \gg_{\omega}$

Now, substituting equations (7) and (8) into the equation (9), we obtain,

$\left.\omega \ll \hat{a}_{\kappa \uparrow}, \hat{a}_{\kappa \uparrow}^{\dagger} \gg=1+\epsilon_{\kappa} \ll \hat{a}_{\kappa \uparrow}, \hat{a}_{\kappa \uparrow}^{\dagger} \gg-\sum_{k k^{\prime}}\left(V_{p h}+V_{e l}-V_{c}\right) \ll \hat{a}_{-\kappa \downarrow}^{\dagger} \hat{a}_{k^{\prime} \downarrow} \hat{a}_{-k^{\prime} \uparrow}, \hat{a}_{\kappa \uparrow}^{\dagger}{ }_{\kappa \uparrow}\right\rangle$

Decoupling higher order Green's function into lower order Green's function, we get, 
$\ll \hat{a}_{-\kappa \downarrow}^{\dagger} \hat{a}_{k^{\prime} \downarrow} \hat{a}_{-k^{\prime} \uparrow}, \hat{a}_{\kappa \uparrow}^{\dagger} \gg=\left\langle\hat{a}_{k^{\prime} \downarrow} \hat{a}_{-k^{\prime} \uparrow}>\left\langle<\hat{a}_{-\kappa \downarrow}^{\dagger}, \hat{a}^{\dagger}{ }_{\kappa \uparrow} \gg\right.\right.$

Substituting equation (11) into equation (10), we get,

$$
\left(\omega-\epsilon_{\kappa}\right) \ll \hat{a}_{\kappa \uparrow}, \hat{a}_{\kappa \uparrow}^{\dagger} \gg=1-\sum_{k k^{\prime}}\left(V_{p h}+V_{e l}-V_{c}\right)<\hat{a}_{k^{\prime} \downarrow} \hat{a}_{-k^{\prime} \uparrow}>\ll \hat{a}_{-\kappa \downarrow}^{\dagger}, \hat{a}_{\kappa \uparrow}^{\dagger} \gg
$$

Let the superconducting order parameter $(\Delta)$ be given by,

$$
\Delta=\sum_{k k^{\prime}}\left(V_{p h}+V_{e l-p h}-V_{c}\right)<\hat{a}_{k^{\prime} \downarrow} \hat{a}_{-k^{\prime} \uparrow}>
$$

Thus, the equation of motion can be expressed as,

$\left(\omega-\epsilon_{\kappa}\right) \ll \hat{a}_{\kappa \uparrow}, \hat{a}^{\dagger}{ }_{\kappa \uparrow} \gg=1-\Delta \ll \hat{a}_{-\kappa \downarrow}^{\dagger}, \hat{a}^{\dagger}{ }_{\kappa \uparrow} \gg$

Following similar procedures as above, the expression for the correlation, $\ll \hat{a}_{-\kappa \downarrow}^{\dagger}, \hat{a}_{\kappa \uparrow}^{\dagger} \gg$ is obtained to be,

$\omega \ll \hat{a}_{-\kappa \downarrow}^{\dagger}, \hat{a}^{\dagger}{ }_{\kappa \uparrow} \gg=-\epsilon_{-\kappa} \ll \hat{a}_{-\kappa \downarrow}^{\dagger}, \hat{a}_{\kappa \uparrow}^{\dagger} \gg-\sum_{k k^{\prime}}\left(V_{p h}+V_{e l}-V_{c}\right)<\hat{a}_{k \uparrow}^{\dagger}, \hat{a}_{-k \downarrow}^{\dagger}>\ll \hat{a}_{\kappa \uparrow}, \hat{a}_{\kappa \uparrow}^{\dagger} \gg$

Now, let the superconducting gap parameter $\left(\Delta^{*}\right)$ be given by,

$\Delta^{*}=\sum_{k k^{\prime}}\left(V_{p h}+V_{e l}-V_{c}\right)<\hat{a}_{k \uparrow}^{\dagger}, \hat{a}_{-k \downarrow}^{\dagger}>$

For $\epsilon_{\kappa}=\epsilon_{-\kappa}$, and $\Delta=\Delta^{*}$ (for real values), we obtain,

$\left(\omega+\epsilon_{\kappa}\right) \ll \hat{a}_{-\kappa \downarrow}^{\dagger}, \hat{a}_{\kappa \uparrow}^{\dagger} \gg=-\Delta \ll \hat{a}_{\kappa \uparrow}, \hat{a}_{\kappa \uparrow}^{\dagger} \gg$

Now, substituting equation (12) into equation (14), the equation of motion becomes,

$\ll \hat{\mathrm{a}}_{-\kappa \downarrow}^{\dagger}, \hat{\mathrm{a}}^{\dagger}{ }_{\kappa \uparrow} \gg=\frac{-\Delta}{\omega^{2}-\left(\epsilon_{\kappa}^{2}+\Delta^{2}\right)}$

Thus, the superconductivity energy order parameter $(\Delta)$ becomes,

$\Delta=\frac{U}{\beta} \sum_{\kappa k^{\prime}} \frac{-\Delta}{\omega^{2}-\left(\epsilon_{\kappa}^{2}+\Delta^{2}\right)}$

Where, $U=V_{p h}+V_{e l}-V_{c}$ and $\beta=\frac{1}{k_{B} T}, k_{B}$ being the Boltzmann constant.

Now, by changing summation into integration and introducing the density of states at the Fermi level, $\mathrm{N}(0)$, we get,

$\Delta=-\frac{1}{\beta} \int_{-\epsilon_{F}}^{\infty} N(0) U \frac{\Delta}{\left(\omega^{2}-\left(\epsilon_{k}{ }^{2}+\Delta^{2}\right)\right.} d \epsilon$

Now, changing $\omega \rightarrow \mathrm{i} \omega_{\mathrm{n}}$, by using the Matsubara frequency (Zubarev, 1960), $\omega_{n}=(2 n+1) \frac{\pi}{\beta}$, we get,

$\Delta=2 N(0) U \beta \sum_{n} \int_{0}^{\hbar \omega_{e l-p h}}\left[\frac{\Delta}{(2 n+1)^{2} \pi^{2}+\beta^{2} E^{2}}\right] d \epsilon$,

Where, $E^{2}=\epsilon_{\kappa}^{2}+\Delta^{2}$

Using the relation,

(C) CNCS, Mekelle University 
$\frac{1}{2 x} \tanh \left(\frac{x}{2}\right)=\sum_{n=-\infty}^{+\infty} \frac{1}{(2 n+1)^{2} \pi+x^{2}}$

Now, we can write equation (18) as,

$\Delta=2 N(0) U \beta \int_{0}^{\hbar \omega_{e l-p h}} \Delta \frac{1}{2 \beta E} \tanh (\beta E / 2) d E$

Let $\lambda=N(0) U$, thus equation (19) becomes,

$\frac{1}{\lambda}=\int_{0}^{\hbar \omega_{e l-p h}} \frac{1}{E} \tanh \left(\frac{E}{2 k_{B} T_{C}}\right) d E$

Let $\mathrm{x}=\frac{E}{2 k_{B} T_{C}}$, which implies, $d E=2 k_{B} T_{c} d x$

Hence, equation (20) becomes,

$\frac{1}{\lambda} \approx \ln \left(1.14 \frac{\hbar \omega_{e l-p h}}{k_{B} T_{C}}\right)$

From which we get,

$$
T_{C}=\frac{1.14 \hbar \omega_{e l-p h}}{k_{B}} \exp \left(-\frac{1}{N(0)\left(V_{p h}+V_{e l}-V_{C}\right)}\right)
$$

Assuming that, $\frac{\hbar}{k_{B}} \approx 1$ and $\omega_{e l-p h} \approx\left(\omega_{e l}\right)^{r_{e l}}\left(\omega_{p h}\right)^{r_{p h}}$, equation (22) becomes,

$T_{C}=1.14\left(\omega_{e l}\right)^{r_{e l}}\left(\omega_{p h}\right)^{r_{p h}} \exp \left(-\frac{1}{\lambda_{p h}+\lambda_{e l}-\mu^{*}}\right)$

where $N(0) V_{p h}=\lambda_{p h}$ is the phonon-exciton coupling constant, $N(0) V_{e l}=\lambda_{e l}$ is the electron (hole)-exciton coupling constant, $N(0) V_{c}=\mu *$ is the parameter arising from Coulomb repulsion, $\omega_{p h}$ and $\omega_{e l}$ are respectively energies of phononic and electronic excitation or cutoff frequencies in temperature units, $r_{e l}=\frac{\lambda_{e l}}{\lambda_{p h}+\lambda_{e l}}$ and $r_{p h}=\frac{\lambda_{p h}}{\lambda_{p h}+\lambda_{e l}}$.

If $\mu^{*}=0$, that is, if we assume that there is no screened repulsion, then equation (23) becomes,

$$
T_{C}=1.14\left(\omega_{e l}\right)^{r_{e l}}\left(\omega_{p h}\right)^{r_{p h}} \exp \left(-\frac{1}{\lambda_{p h}+\lambda_{e l}}\right)
$$

The theoretical values of the transition temperature $\left(\mathrm{T}_{\mathrm{C}}\right)$ of $B a_{1-x} K_{x} F e_{2} A s_{2}$, for $\lambda_{\mathrm{ph}}=0.3$, $\omega_{\mathrm{ph}}=300 \mathrm{~K}, \omega_{\mathrm{el}}=4000 \mathrm{~K}$ (in temperature unit) (Sinha, 2014) and for different values of $\lambda_{\mathrm{el}}$ (say, $\lambda_{\text {el }}=0.072$ ), is obtained to be, $\mathrm{T}_{\mathrm{C}}=38.4 \mathrm{~K}$, which is in good agreement with the experimental value, $\mathrm{T}_{\mathrm{C}}=38 \mathrm{~K}$ for $B a_{1-x} K_{x} F e_{2} A s_{2}$ (Rotter, et al., 2008). 


\subsection{Temperature dependent superconducting energy gap}

To obtain the temperature dependent superconducting energy gap, we use the same techniques as above to solve the integral, given by,

$\frac{1}{\lambda}=\int_{0}^{\hbar \omega_{e l-p h}}\left(\frac{1}{\left.\sqrt{\epsilon^{2}+\Delta^{2}}\right)} \tanh \left(\beta \frac{\left.\sqrt{\epsilon^{2}+\Delta^{2}}\right)}{2}\right)\right) d \epsilon$

After a couple of steps we obtain,

$T_{C}=\ln 1.14 \frac{\hbar e l-p h}{k_{B} T}-\Delta^{2}\left(\frac{1}{\pi k_{B} T}\right)^{2}(1.05)+\cdots$

But from the BCS model, $\frac{1}{\lambda}=\ln \left(1.14 \frac{\hbar \omega_{\mathrm{b}}}{\mathrm{k}_{\mathrm{B}} \mathrm{T}_{\mathrm{c}}}\right)$, where, as $T \rightarrow T_{c}, \Delta \rightarrow 0$.

Thus, equation (25) can be simplified and obtain,

$\ln \left(T / T_{c}\right)=-\Delta^{2}\left(\frac{1}{\pi k_{B} T_{c}}\right)^{2}(1.05)+\cdots$

Using the relation $\ln (1-x)=-x-\frac{x^{2}}{2}+---$, and rearranging, we obtain,

$\Delta(\mathrm{T})=3.06 \mathrm{k}_{\mathrm{B}} \mathrm{T}_{\mathrm{c}}\left(1-\frac{\mathrm{T}}{\mathrm{T}_{\mathrm{c}}}\right)^{\frac{1}{2}}$

Equation (26) demonstrates how the superconducting order parameter, $\Delta(\mathrm{T})$ varies with temperature and is analogous to the BCS model.

From the BCS theory, $\Delta(0) \approx 2 k_{B} T_{C}$, and using the experimental value, $T_{C}=38 \mathrm{~K}$, for $B a_{1-x} K_{x} F e_{2} A s_{2}$, the superconducting order parameter at zero temperature, $\Delta(0)$, becomes, $\Delta(0)=9.42 \mathrm{meV}$.

\section{RESULTS AND DISCUSSION}

In this section, we analyzed the results which are obtained using the model Hamiltonian developed. We obtained the expressions for the superconducting order parameter $(\Delta)$ and superconducting transition temperature $\left(\mathrm{T}_{\mathrm{C}}\right)$.

Now, by using equation (24) and the experimental value of $\mathrm{T}_{\mathrm{C}}$ for the iron based superconductor $\mathrm{Ba}_{1-x} \mathrm{~K}_{x} \mathrm{Fe}_{2} \mathrm{As}_{2}$ and plausible approximations for other parameters (such as for $\lambda_{\mathrm{ph}}=0.3$, $\left.\omega_{\mathrm{ph}}=300 \mathrm{~K}, \omega_{\mathrm{el}}=4000 \mathrm{~K}\right)$, we plotted the transition temperature $\left(\mathrm{T}_{\mathrm{C}}\right)$ versus electron coupling constant $\left(\lambda_{\mathrm{el}}\right)$ as shown in figure 1. 


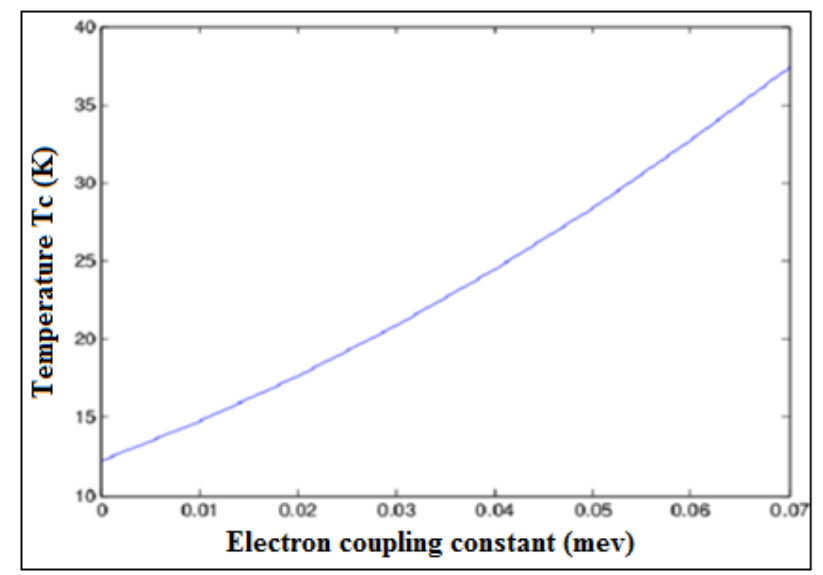

Figure 1. Electron coupling constant $\left(\lambda_{\mathrm{el}}\right)$ versus superconducting temperature $\left(\mathrm{T}_{\mathrm{C}}\right)$ for $B a_{1-x} K_{x} F e_{2} A s_{2}$ superconductor.

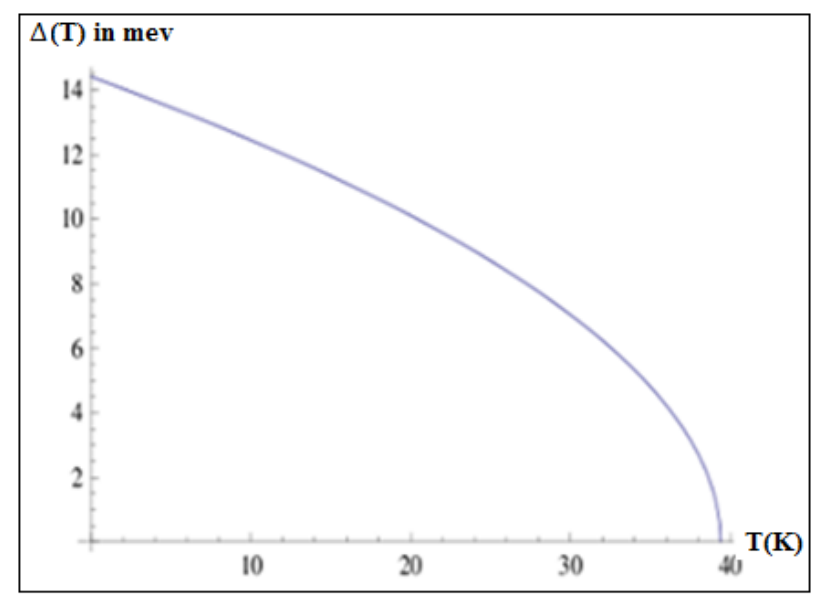

Figure 2. Transition temperature $\left(\mathrm{T}_{\mathrm{C}}\right)$ versus superconducting order parameter $(\Delta)$ for $B a_{1-x} K_{x} F_{2} A s_{2}$ superconductor.

As can be seen from figure 1, when the electron coupling constant $\left(\lambda_{\mathrm{el}}\right)$ increases the superconducting transition temperature $\left(\mathrm{T}_{\mathrm{C}}\right)$ increases and vice versa. Furthermore, by using equation (26) and the experimental values, $\mathrm{T}_{\mathrm{C}}=38 \mathrm{~K}$, for $B a_{1-x} K_{x} F e_{2} A s_{2}$, we plotted the transition temperature $\left(\mathrm{T}_{\mathrm{C}}\right)$ versus superconducting order parameter $(\Delta)$ as shown in figure 2 .

As can be seen from figure 2, when the temperature increases the superconducting order parameter decreases and vanishes at the transition temperature $\left(\mathrm{T}_{\mathrm{C}}\right)$ of $B a_{1-x} K_{x} F e_{2} A s_{2}$.

\section{CONCLUSION}

In this investigation, by considering the phonon-exciton combined mechanism and by using the experimental and plausible theoretical values of the parameters in the obtained expressions, we plotted phase diagrams of superconducting transition temperature $\left(T_{\mathrm{C}}\right)$ versus electron coupling 
constant and superconducting transition temperature $\left(T_{\mathrm{C}}\right)$ versus superconducting parameter. The obtained results are in broad agreement with previous findings (Sinha, 2014).

\section{REFERENCE}

Allender, D., Bray, J \& Bardeen, J. 1973. Model for an exciton Mechanism of Superconductivity. Phys. Rev., 7: 1020.

Bardeen, J., Cooper, L. N \& Schrieffer, J. R. 1957. Theory of Superconductivity. Phys. Rev., 108: $1175-1204$.

Bednorz, J.G \& Müller, K. A. 1986. Possible high temperature superconductivity in the LaBaCuO system. Z Phys. B., 64: 189-193.

Hsu, F.C., Luo, J.Y., Yen, K.W., Chen, T.K., Huang, T.W., Wu, P.M., Lee, Y.C., Huang, Y.L., Chu, Y.Y., Yan, D.C \& Wu, M.K. 2008. Superconductivity in the PbO-type structure $\alpha$ FeSe, Proceedings of the National Academy of Sciences, 105: 14262-14264.

Kamihara, Y., Watanabe, T., Hirano, M \& Hosono, H. 2008. Iron-Based Layered Superconductor $\mathrm{La}\left(\mathrm{O}_{1-\mathrm{x}} \mathrm{F}_{\mathrm{x}}\right) \mathrm{FeAs}(\mathrm{x}=0.05-0.12)$ with $\mathrm{T}_{\mathrm{C}}=26 \mathrm{~K}, J$. Am. Chem. Soc., 130: 3296.

Onnes, H. K. 1911. Akademiavan Wetenschappen (Amsterdam), 14:113.

Rotter, M., Tegel, M \& Johrendt, D. 2008. Superconductivity at 38K in the Iron Arsenide,Phys. Rev. Let., 101:107006.

Singh, P \& Sinha, K.P. 1990. A possible mechanism of high $\mathrm{T}_{\mathrm{C}}$ superconductivity involving biexcitons. J. Sol. Sta. Commun., 73:45.

Sinha, K.P. 2014. The mechanisms of some High Temperature and Novel Superconductors. Proc. Indian Natn Sci Acad., 80:23-35.

Wang, C., Li, L., Chi, S., Zhu, Z., Ren, Z., Li, Y., Wang, Y., Lin, X., Luo, Y., \& Jiang, S. 2008 a. Thorium doping induced superconductivity up to $56 \mathrm{~K}$ in $\mathrm{Gd}_{1-\mathrm{x}} \mathrm{Th}_{\mathrm{x}} \mathrm{FeAsO}$. EuroPhys. Lett., 83:67006.

Wang, X.C., Liu, Q.Q., Lv, Y.X., Gao, W.B., Yang, L.X., Yu, R.C., Li, F.Y. \& Jin,C.Q. $2008 b$. The superconductivity at $18 \mathrm{~K}$ in LiFeAs system. Solid State Commun., 148:538-540.

Zubarev, D. N. 1960. Double -Time Green Functions in Statistical Physics. Soviet Physics. Uspekhi, 3:320. 